\title{
Retinoid metabolism: a balancing act
}

Thomas Perlmann

Nature Genet. 31, 7-8 (2002).

Two errors were introduced while preparing this article for press. They were introduced after the author provided his corrections to the proofs, and so he did not have an opportunity to correct the errors. In two places, Aldh1a2, an aldehyde dehydrogenase, is referred to incorrectly as an alcohol dehydrogenase. This occurs in the first sentence of the strapline and in the last sentence of the fourth paragraph beginning "Although the picture remains incomplete..." The corrected version of this article is available in PDF format on the Nature Genetics website. 